\title{
Spectroscopy in ep collisions at HERA
}

\section{Ozerov ${ }^{\mathrm{a}}$ (on behalf of the H1 and ZEUS Collaborations)}

${ }^{a}$ A.I. Alikhanov Institute for Theoretical and Experimental Physics, Bolshaya Cheremushkinskaya 25, 117218 Moscow, Russia, and Deutsches Elektronen-Synchrotron, Notkestrasse 85, 22607 Hamburg, Germany

Recent spectroscopy studies performed in high energy ep collisions by the H1 and ZEUS Collaborations are described. The status of the searches for the strange pentaquark, the $\theta^{+}$, is presented as well as results on searches for further exotic states such as the $\Sigma^{--}$. The evidence for the observation of a pentaquark state containing the charmed quark, the $\theta_{c}$, is discussed and the result is given on searches for glueball candidates at HERA.

During the first stage of operation of the unique ep collider HERA, the two Collaborations $\mathrm{H} 1$ and ZEUS collected data samples corresponding to more than $100 \mathrm{pb} \mathrm{b}^{-1}$ each. Good tracking detectors allow the identification of various resonances via their charged particle decay modes. One study was performed by the ZEUS Collaboration using events with at least two well identified $K_{s}^{0}$, detected via its decay to two charged pions. Such a $K_{s}^{0} K_{s}^{0}$ system is of particular interest since it is expected to couple to scalar and tensor glueballs [1]. Lattice QCD calculations predict [2] the existence of a scalar glueball with a mass of $1730 \pm 100 \mathrm{MeV}$, close to the observed $f_{0}(1710)$ state. The details of the event selection and analysis can be found in [3]. Figure 1] shows the measured $K_{s}^{0} K_{s}^{0}$ invariant mass spectrum. In the region above $1500 \mathrm{MeV}$, two narrow resonances can be seen. The lower mass state has a fitted mass of $1537_{-8}^{+9} \mathrm{MeV}$ and a width of $50_{-22}^{+34} \mathrm{MeV}$, the higher mass state has a fitted mass of $1726 \pm 7 \mathrm{MeV}$ and a width of $38_{-14}^{+20} \mathrm{MeV}$. The first state is in a good agreement with the $f_{2}^{\prime}(1525)$ state, while the second is consistent with the glueball candidate $f_{0}(1710)$. It is interesting to note that the selected $K_{s}^{0}$-pair originated predominantly from the region where sizeable initial state gluon radiation may be expected.

The hot topic in spectroscopy today is the discussion of the possible existence of a pentaquark state with extremely narrow width and low mass. Such states were predicted in [4]. The ZEUS Collaboration analysed the system $K_{s}^{0} p(\bar{p})$, where $K_{s}^{0}$ was again identified via its decay to charged pions and the (anti)proton by measuring the charged particle ionization. Details can be found in [ 5]. Figure 2] shows the $K_{s}^{0} p(\bar{p})$ invariant mass for $Q^{2}>20 \mathrm{GeV}^{2}$, where $Q^{2}$ is the squared momentum transfer between lepton and nucleon. The fit of two Gaussians on top of a continuous background is shown on the picture. The first Gaussian describes the narrow peak at $1522 \mathrm{MeV}$, while the second Gaussian, near $1470 \mathrm{MeV}$, represents the complicated background shape in this region, which may be due to the broad resonance $\Sigma(1480)$. The peak position obtained from the fit is $1521.5 \pm 1.5$ (stat. $)_{-1.7}^{+2.8}$ (syst.) MeV. The Gaussian width was found to be $6.1 \pm 1.6$ 

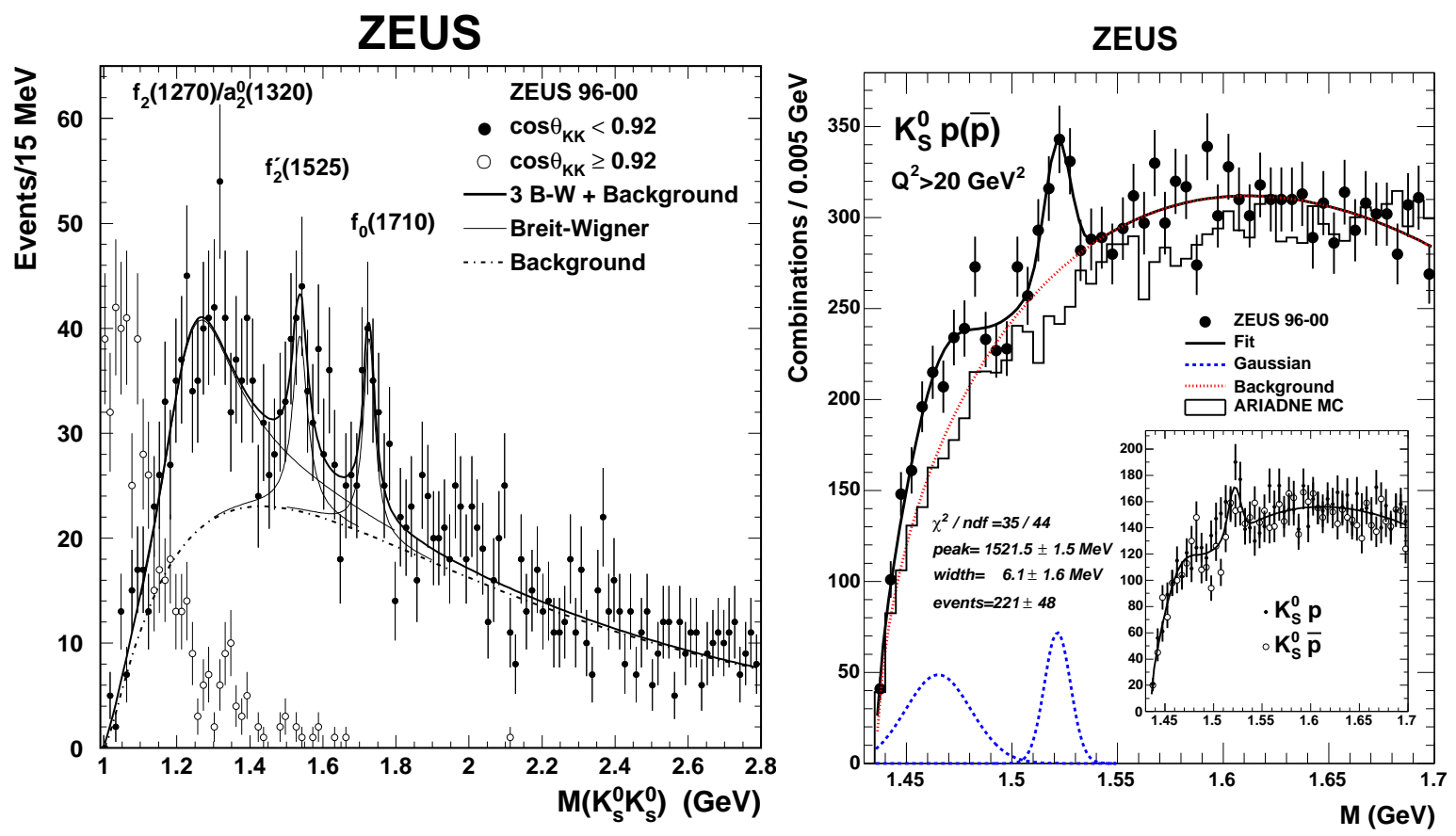

Figure 1. The $K_{s}^{0} K_{s}^{0}$ invariant-mass spectrum for $K_{s}^{0}$ pair candidates with $\cos \theta_{K_{s}^{0} K_{s}^{0}}<$ 0.92 (filled circles). The thick solid line is the result of a fit using three Breit-Wigner (thin solid lines) and a background function (dotted-dashed line). The $K_{s}^{0}$ pair candidates that fail the $\cos \theta_{K_{s}^{0} K_{s}^{0}}<0.92$ cut are also shown (open circles).

Figure 2. Invariant mass for the $K_{s}^{0} p(\bar{p})$ resonance. The histogram depicts the predictions from the Monte Carlo simulation and is normalized to the data in the region above $1650 \mathrm{MeV}$. The inset shows the measurements for the $K_{s}^{0} p$ (black dots) and $K_{s}^{0} \bar{p}$ (open circles) candidates separately.

$\mathrm{MeV}$, compatible with the experimental resolution of $2 \mathrm{MeV}$. The fit of the Breit-Wigner, convoluted with a Gaussian with a width fixed to the experimental resolution, gives a signal BW-width of $8 \pm 4$ (stat.) $\mathrm{MeV}$. The number of events obtained from the fit is $221 \pm 48$, which corresponds to the statistical significance of $4.6 \sigma$. The fit of the $K_{s}^{0} \bar{p}$ combinations gives $96 \pm 34$ events which agrees with the signal extracted from the $K_{s}^{0} p$ channel. If the $K_{s}^{0} p$ signal corresponds to the $\theta^{+}$pentaquark observed by several other experiments, this measurement provides the first evidence for the antiparticle $\theta^{-}$with the quark content of $\bar{u} \bar{u} \bar{d} \bar{d} s$. Figure 3 shows the $\theta$ production cross section for $Q^{2}>Q_{\text {min }}^{2}$, measured in the kinematic region $0.04<y<0.95, P_{T}(\theta)>0.5 \mathrm{GeV}$ and $|\eta(\theta)|<1.5$ (where $y$ is the lepton inelasticity), as a function of $Q_{\min }^{2}$. The cross section ratio $R$ of $\theta$ to the well-known $\Lambda(1116)$ baryonic state was measured to be $R=\left(4.2 \pm 0.9_{-0.9}^{+1.2}\right) \%$ for $Q^{2}>20 \mathrm{GeV}^{2}$. Figure 4 shows $R$ for several $Q_{\text {min }}^{2}$ values.

The $p p$ fixed target NA49 Collaboration reported [ 6] observation of the $\Xi$ multiplet pentaquark candidates $\Xi_{3 / 2}^{--}$and $\Xi_{3 / 2}^{0}$, predicted in [4] together with the $\theta^{+}$pentaquark. The narrow resonances were observed in $\Xi \pi$ combinations at masses $\approx 1862 \mathrm{MeV}$. ZEUS performed [7] a search for such states. No evidence of a signal at about $1860 \mathrm{MeV}$ was 


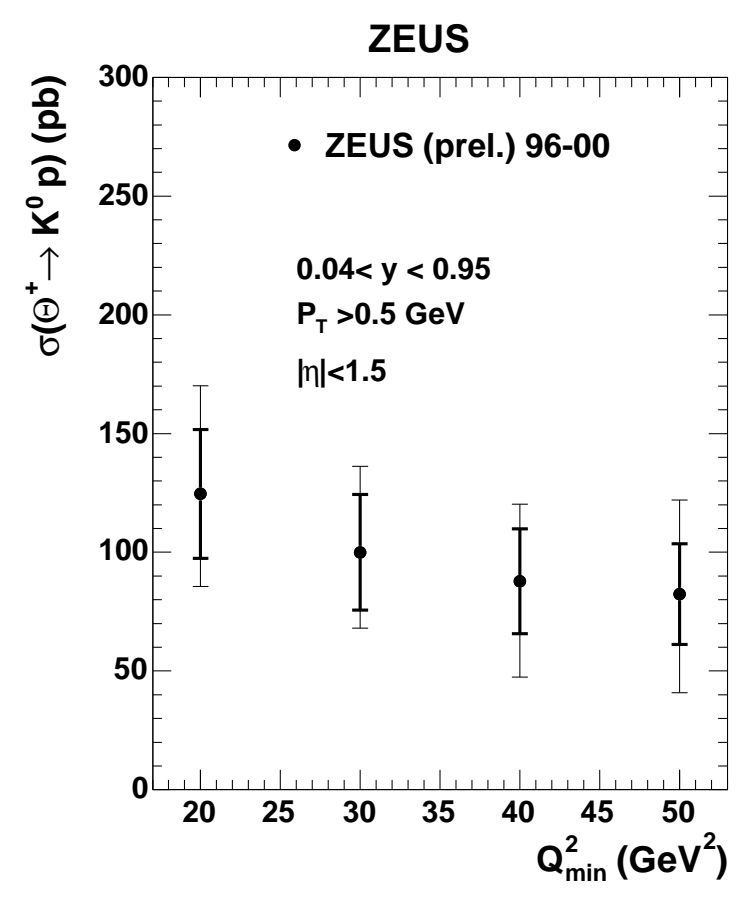

Figure 3. Visible cross sections for the $\theta^{ \pm}$baryon decaying to $K_{s}^{0} p(\bar{p})$ as a function of $Q_{m i n}^{2}$.

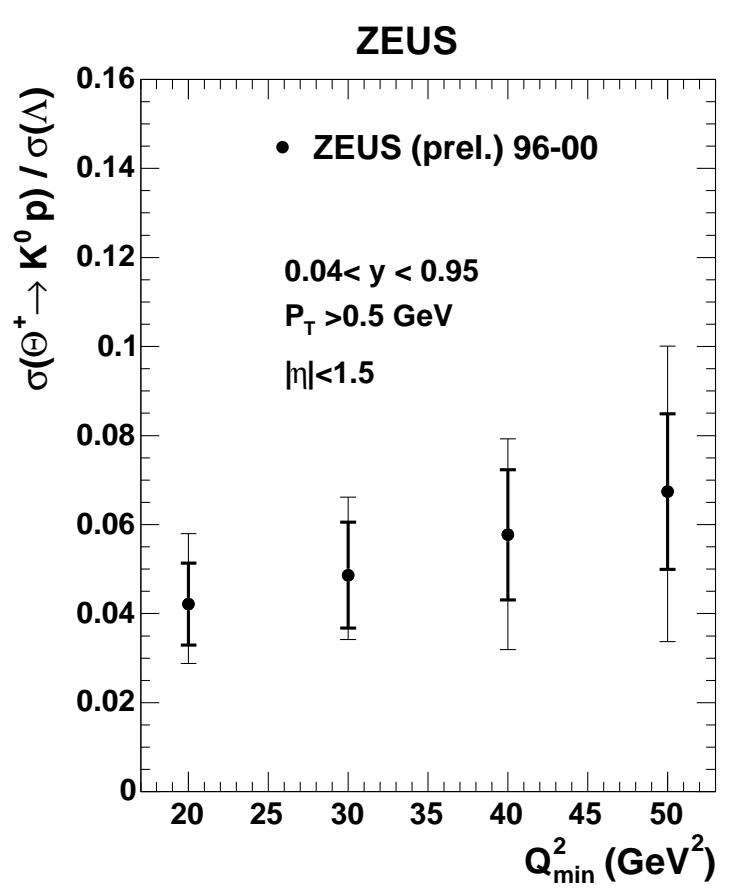

Figure 4. Cross sections ratio $R=\sigma\left(\theta^{+} \rightarrow K_{s}^{0} p\right) / \sigma(\Lambda)$ as a function of $Q_{\min }^{2}$.

found in any $\Xi \pi$ combination, while a clean $\Xi^{0}(1530)(\rightarrow \Xi \pi)$ is seen with a significance of $\approx 4.8 \sigma$. The discrepancy in the results of the two experiments can be attributed to the fact that ZEUS made the search in the central rapidity region, while NA49 covers the forward rapidity region.

The existence of the strange pentaquark $\theta^{+}=u u d d \bar{s}$ implies that a pentaquark with the charmed quark, $u$ udd $\bar{c}$, could also exist. If the mass of such a state is above the sum of the mass of a $D^{*}$ and a proton, it may decay to $D^{* \pm} p^{\mp}$. The H1 Collaboration found [ 8 ] a narrow signal in the $D^{* \pm} p^{\mp}$ invariant mass at $3.1 \mathrm{GeV}$ with a width consistent with the detector resolution. The $D^{*}$ mesons were identified using the $D^{* \pm} \rightarrow D^{0} \pi^{ \pm} \rightarrow\left(K^{\mp} \pi^{ \pm}\right) \pi^{ \pm}$ decay channel. A clean signal is seen in DIS $\left(Q^{2}>1.0 \mathrm{GeV}^{2}\right)$ and, independently, in the photoproduction sample $\left(Q^{2}<1.0 \mathrm{GeV}^{2}\right)$, as shown in figure 5 . The probability of the background to fluctuate to the signal in the DIS sample was estimated to be less than $4 \cdot 10^{-8}$ which corresponds to $5.4 \sigma$ in terms of Gaussian standard deviations. The ZEUS Collaboration made a similar search for such a state [9]. The ZEUS $D^{*}$ mesons were reconstructed using two decay channels of the $D^{0}, D^{0} \rightarrow K^{\mp} \pi^{ \pm}$and $D^{0} \rightarrow K^{\mp} \pi^{ \pm} \pi^{+} \pi^{-}$. The integrated luminosity of the ZEUS data sample is 1.7 times larger than that of the H1 data sample. No narrow signal was observed in either of the $D^{0}$-decay modes. The selection criteria were different in the two analyses, resulting in different phase space region explored and in a larger yield of reconstructed $D^{*}$ in the ZEUS investigation. The ZEUS Collaboration presented also results in the region similar to the H1 study and found no indication of a narrow resonance, neither in the DIS nor in the photoproduction sample 

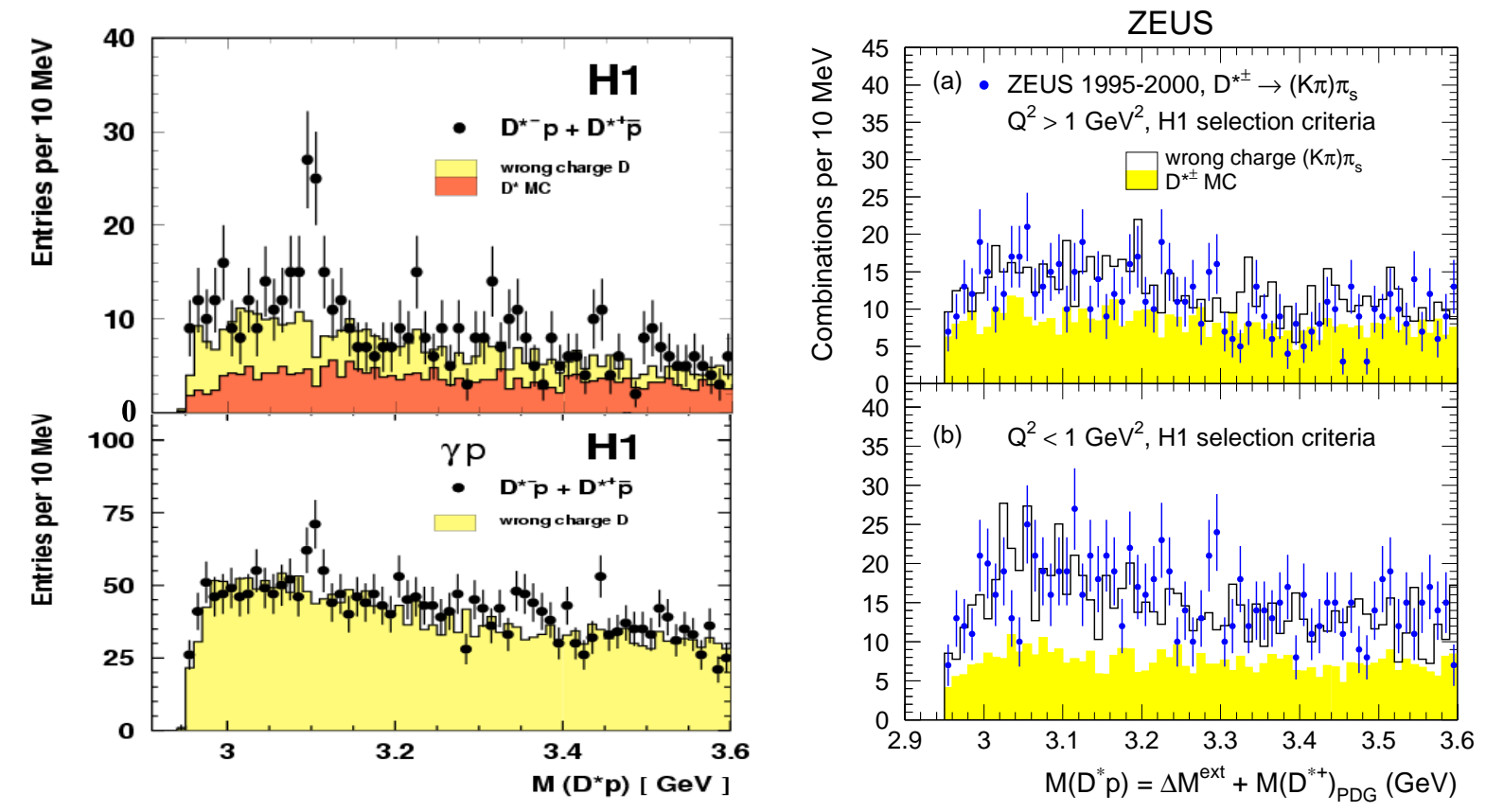

Figure 5. Invariant mass of the opposite signs $D^{*} p$ combinations for the DIS (top) and photoproduction (bottom) data samples of the H1 Collaboration.

Figure 6. Invariant mass of the opposite signs $D^{*} p$ combinations for the DIS (top) and photoproduction (bottom) data samples of the ZEUS Collaboration with selection criteria similar to $\mathrm{H} 1$.

(see figure 6). New data from HERA-II will help to resolve this discrepancy in the results of the two experiments.

It is a pleasure to thank the organizers for the warm and joyful atmosphere in a most interesting and remarkably well prepared conference. I also wish to thank my colleagues in $\mathrm{H} 1$ and ZEUS, for providing the data and results presented in this report and for all their help given to me.

\section{REFERENCES}

1. S. Godfrey and J. Napolitano, Rev. Mod. Phys. 71 (1999) 1411;

E. Klempt, Proc. of Zuoz 2000, Phenomenology of gauge interactions (2000) 61.

2. C. J. Morningstar and M. J. Peardon, Phys. Rev. D 60 (1999) 034509;

C. Michael and M. Teper, Nucl. Phys. B 314 (1989) 347.

3. S. Chekanov et al. [ZEUS Coll.], Phys. Lett. B 578 (2004) 33.

4. D. Diakonov, V. Petrov and M. V. Polyakov, Z. Phys. A 359 (1997) 305.

5. S. Chekanov et al. [ZEUS Coll.], Phys. Lett. B 591 (2004) 7;

A. Raval [for the ZEUS Coll.], Presented at ICHEP 2004.

6. C. Alt et al. [NA49 Coll.], Phys. Rev. Lett. 92 (2004) 042003.

7. S. Chekanov et al. [ZEUS Coll.], DESY 05-018.

8. A. Aktas et al. [H1 Coll.], Phys. Lett. B 588 (2004) 17.

9. S. Chekanov et al. [ZEUS Coll.], Eur. Phys. J. C 38 (2004) 29. 